Entries for the prize should be presented in the form of an essay or dissertation, with accompanying tables and figures. A collection of articles will not be accepted as a submission for the prize, but previously published work may be included in the submission if it is incorporated into the essay or dissertation.

Recipients of the prize will be invited to present a report at the Scientific Meeting of the Child and
Adolescent Psychiatry Specialist Section which is held in the Autumn.

The examiners of the Gillian Page Research Prize will be nominated by the Council of the Royal College of Psychiatrists. No prize will be awarded if a sufficient standard is not reached.

Entries for the prize should be submitted to the Dean by 31 March 1989.

\title{
The College Appeal
}

The following members have generously contributed to the College Appeal Fund for the Research Unit since July 1987.

Dr A. J. Abraham Dr R. C. Adams Dr K. A. Akuffo-Akoto

Dr E. A. Alderson

Dr R. Anderson

Dr P. A. Ashurst

Prof T. Baasher

Dr S. Bagir-Husain

Dr V. Bailey

Dr J. A. Baird

Dr A. F. Barker

Dr J. L. Barton

Dr L. A. Bates

Dr K. Bergmann

Dr J. Bird

Dr P. D. Black

Dr S. Bloch

Dr H. R. Bond

Dr W. R. Breakey

Dr P. W. Brooks

Dr D. J. Brooksbank

Dr J. J. Brophy

Dr D. P. K. Brown

Dr H. Bullard

Dr M. Cahill

Dr M. T. G. T. Campbell

Dr R. J. Cantrell

Dr H. M. Capes

Dr W. J. Charles

Dr B. Chester

Dr I. G. Christie

Dr I. Clark
Dr M. Cleary

Dr G. N. Conacher

Dr C. C. H. Cook

Dr I. B. Cookson

Dr M. A. Cooper

Dr M. H. Cooper

Prof J. A. Corbett

Dr C. M. Corser

Prof A. D. Cox

Dr J. Crisp

Dr W. M. Croke

Dr E. Crouch

Col R. G. Davies

Dr R. H. Davies

Dr S. L. Davies

Dr T. G. Davies

Dr A. K. De

Dr D. M. Dickens

Dr H. A. Dickinson

Prof A. Diji

Dr J. Donnelly

Dr E. M. Dooley

Dr A. Duncan

Dr T. L. Dunn

Dr J. M. Eagles

Dr J. Guy Edwards

Dr O. E. A. F. El-Rufaie

Dr M. Faulk

Dr L. Fernando

Dr I. N. Ferrier

Dr M. Ford

Dr G. C. Forrest
Dr D. G. Fowlie

Dr M. F. Foyle

Dr W. Fraser

Dr A. M. Gaitonde

Dr D. J. Galbraith

Dr P. L. G. Gallwey

Dr S. M. Gibson

Dr D. C. Gill

Dr J. E. Gillespie

Dr A. Gordon

Dr J. Gordon

Dr A. Graham

Prof P. J. Graham

Dr P. H. Groves

Dr J. H. Gunn

Dr M. A. Halim

Dr Z. Hall

Dr C. M. Harding

Dr E. H. Hare

Dr D. J. Harris

Dr G. Harrison

Dr C. F. Herridge

Dr M. Hinchcliffe

Prof S. R. Hirsch

Dr F. Hocking

Dr R. Hoffman

Dr H. M. Holden

Dr D. Hollander

Dr S. A. Humphreys

Dr P. V. Hunt

Dr E. A. Hutchesson

Dr G. M. Hyams
Dr H. Inceman

Dr R. C. Ingrey-Senn

Dr C. F. Iribarne

Dr S. S. M. Jawad

Jordan Psychiatric Association *Dr F. A. Judelsohn Prof L. B. Kalinoskwy Dr K. M. G. Keddie Dr A. M. P. Kellam

Dr R. I. Kennedy

Dr S. H. Kennedy

Dr T. A. Kerr

Dr J. Kinross-Wright

Prof I. Kolvin

Dr L. M. Kremer

Dr J. B. Labia

Dr P. W. W. Leach

Dr A. S. Lee

Dr A. H. Leighton

Dr J. T. Leyberg

Dr M. W. Leyshon

Dr L. I. Liebling

Dr C. S. Lindsay

Dr W. H. Lo

Dr S. H. Lorbeer

Dr E. G. Lucas

Dr H. A. McClelland

Dr R. McVitie

Dr S. MacKeith

Dr M. Mackenzie

Dr D. S. Macphail 
Dr J. S. Madden Sir Paul Mallinson Dr C. Mani Dr W. D. Mann Dr H. J. Marrable Dr J. A. S. Marriott Dr E.S. Martin Dr P. Melia Prof J. E. Meyer Dr W. I. Mikhail Dr J. Milne Dr C. E. Monaghan Dr D. H. Morgan Major J. D. Mumford Dr M. Murphy Dr K. N. Murray Dr A. Mylvaganam Dr H.S. Ng Prof A. R. Nicol Dr H.S. Nicolson Dr S. V. Oakes Dr K. O'Gorman Dr J. O'Hanlan Dr P. i. O'Hara Dr B. E. Oliver
Dr C. Oppenheimer Dr J. Parker Dr R. Parker * Dr R. G. Polson Dr A. J. Prange Dr H. A. Prins Dr S. E. Proctor Dr I. G. Pryce Dr E. W. Pugh Dr R. Nada Raja Dr M. Ravey Dr A. Razzak Dr R. W. K. Reeves Dr B. W. Richards Dr D. Richter Dr D. Riley Dr K. J. B. Rix Dr E. Roderic-Evans Dr E. T. Rolston Dr J. D. Russell Dr G. G. C. Rwegellera Dr J. N. Scott Dr O. E. P. Shanks Dr D. Shanley Dr H. E. Sharrard
Dr D. M. Shaw Dr G. Shaw Dunn Dr B. Shorvon Dr C. D. T. Sibisi Dr H. R. Simons Dr R. J. Simpson Dr P. W. Skerrit *Dr C. G. Smith Dr E. D. Smith Dr S. M. Smout Dr J. Sneddon Prof G. F. Solomon Dr P. V. Spencer Dr D. Stastny Prof E. Strömgren Dr P. A. Sugarman Dr S. Sungum-Paliwai Dr R. E. Talbot Dr P. C. Talkington Dr W. Y. K. Tam Dr A. J. Taylor Dr P. J. Taylor Dr D. E. Tesh Dr J. R. Theobalds Dr A. Theodossiadis
Dr M. S. Towse

Dr D. Turkington Dr G. C. Turle Dr E. Tylden Dr S. P. Tyrer Dr D. Veale Dr J. R. Vettraino Mr A. V. Vickery Dr M. M. Walker Dr J. H. Watkin Dr E. Welldon Dr B. Wharton Dr P. B. Whatmore Dr P. D. White Dr D. N. Wilson Dr R. Wilson Dr S. Wolff Dr R. J. Wool Dr J. G. Wright Dr D. Yeldham Dr P. M. Yellowlees Prof K. A. Yonge

We have also received a donation from the friends and relatives of the late Mr A. S. Marwick.

*Drs Polson, Judelsohn and C. G. Smith's names unfortunately were omitted from a previously published list of contributing members, for which we apologise.

If your name has been omitted from this list could you please contact Mrs Chris Gear at the College to ensure that it is included on a future list. 